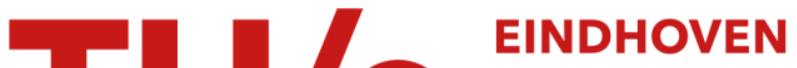 UNIVERSITY OF TECHNOLOGY
}

\section{Fröhlich interaction in InAs/GaAs self-assembled quantum dots}

Citation for published version (APA):

Minnaert, A. W. E., Silov, A. Y., Vleuten, van der, W. C., Haverkort, J. E. M., \& Wolter, J. H. (2001). Fröhlich interaction in InAs/GaAs self-assembled quantum dots. Physical Review B, 63(7), 075303-1/4. [075303]. https://doi.org/10.1103/PhysRevB.63.075303

DOI:

10.1103/PhysRevB.63.075303

Document status and date:

Published: 01/01/2001

\section{Document Version:}

Publisher's PDF, also known as Version of Record (includes final page, issue and volume numbers)

\section{Please check the document version of this publication:}

- A submitted manuscript is the version of the article upon submission and before peer-review. There can be important differences between the submitted version and the official published version of record. People interested in the research are advised to contact the author for the final version of the publication, or visit the $\mathrm{DOI}$ to the publisher's website.

- The final author version and the galley proof are versions of the publication after peer review.

- The final published version features the final layout of the paper including the volume, issue and page numbers.

Link to publication

\section{General rights}

Copyright and moral rights for the publications made accessible in the public portal are retained by the authors and/or other copyright owners and it is a condition of accessing publications that users recognise and abide by the legal requirements associated with these rights.

- Users may download and print one copy of any publication from the public portal for the purpose of private study or research.

- You may not further distribute the material or use it for any profit-making activity or commercial gain

- You may freely distribute the URL identifying the publication in the public portal.

If the publication is distributed under the terms of Article 25fa of the Dutch Copyright Act, indicated by the "Taverne" license above, please follow below link for the End User Agreement:

www.tue.nl/taverne

Take down policy

If you believe that this document breaches copyright please contact us at:

openaccess@tue.nl

providing details and we will investigate your claim. 


\title{
Fröhlich interaction in InAs/GaAs self-assembled quantum dots
}

\author{
A. W. E. Minnaert, ${ }^{*}$ A. Yu. Silov, ${ }^{\dagger}$ W. van der Vleuten, ${ }^{\ddagger}$ J. E. M. Haverkort, and J. H. Wolter \\ Department of Physics, COBRA Interuniversity Research Institute, Eindhoven University of Technology, \\ P.O. Box 513, 5600 MB Eindhoven, The Netherlands
}

(Received 21 December 1999; revised manuscript received 5 June 2000; published 18 January 2001)

\begin{abstract}
The phonon bottleneck in self-assembled InAs/GaAs quantum dots (SAD's) is observed directly in continuous-wave photoluminescence experiments when exciting one GaAs longitudinal optical (LO)-phonon energy above the ground level of the smallest dot. To overcome the phonon bottleneck, selective photoluminescence (PL) experiments are performed and multiple phonon-assisted radiative bands are observed. We found that no real crystal states are involved in the experimentally observed phonon emission. Under nonresonant excitation at $5 \mathrm{~K}$, the SAD's photoluminescence band is centered at $1.315 \mathrm{eV}$. As proven by our photoluminescence experiments at high excitation densities, there are no excited states in such small dots. We interpret the phonon-assisted PL as being due to enhanced Fröhlich interaction between strain-induced polarized excitons in the SAD's and LO phonons. Further experimental support for this model is found from the cleaved-side PL measurements. A light-hole ground state is observed, instead of the theoretically predicted heavy-hole one.
\end{abstract}

DOI: 10.1103/PhysRevB.63.075303

PACS number(s): 78.55.Cr, 71.38.-k

\section{INTRODUCTION}

For the past few years, self-assembled quantum dots (SAD's) have been intensively studied both experimentally and theoretically. So have, among many other features, multiphonon assisted relaxation processes have been reported. ${ }^{1-3}$ They are explained in terms of the intradot electronic transitions, which coincide with the available phonon energies. Besides the experimental effort, recent theoretical papers ${ }^{4-6}$ on quantum dots show an improvement in calculating the strain distribution by, e.g., the valence force-field method. These papers agree well with the explanation of the electronic transitions and phonon processes observed.

However, intersubband transitions cannot explain the multiphonon assisted relaxation processes, which we observed in much smaller dots than reported in Refs. 1-3. Moreover, recent results ${ }^{7}$ show that, even in larger dots with excited states, interlevel relaxation is a point of discussion. The dots discussed in this paper are comparable with those of Ref. 8. They are typically $70-120 \AA$ at the base and 25-40 A high. This makes the electronic structure of our dots completely different compared to the larger dots, since they have just one bound electron state and one bound heavy- and light-hole state. ${ }^{8}$ Besides, the ground states of these smaller dots are already close to the energy levels in the wetting layer, which makes the distinction between any excited state and a wetting layer state impossible.

The multiphonon processes reported here are explained in terms of a strong Fröhlich interaction, in which no real crystal states are involved. ${ }^{9,10}$ The phonon emission is observed under resonant excitation measurements, i.e., exciting a given dot size with the ground-state energy fitting to the excitation energy. This results in up to four phonon emission enhanced bands below the excitation energy. These bands do not show any fine structure. ${ }^{1}$ In this respect, the phonon effects observed in our experiments are comparable with Refs. 2 and 3.

In order to have a strong Fröhlich interaction, a local po- larization has to be present in the dots provided by a nonuniform strain field. An indication for such a nonuniform strain field is found in our polarization-dependent photoluminescence measurements where the light-hole ground state is observed. This is in contradiction with the heavy-hole ground state predicted in most theoretical works. ${ }^{4,5,11}$

Despite the observed effective phonon emission, these dots still display the phonon bottleneck, as we directly obtain from continuous wave (cw) photoluminescence (PL) experiments. When exciting close to the edge of the density of states (DOS) in the wetting layer, the dots are more efficiently populated by emitting longitudinal optical (LO) phonons than by relaxation through the wetting layer. The luminescence of the dots is enhanced on a LO-phonon energy of $\pm 36.0 \mathrm{meV}$ (GaAs-like) and $\pm 70.0 \mathrm{meV}$ (2 $\times$ GaAs-like) below the excitation energy. However, the luminescence is partly suppressed between these two energies as well as above the second LO-phonon band.

\section{EXPERIMENTS}

\section{A. Sample}

The quantum dots are grown on an exactly oriented [001] CrO-doped $\mathrm{GaAs}$ substrate. First a GaAs buffer layer was grown at $630^{\circ} \mathrm{C}$, followed by two monolayers of InAs deposited at $555^{\circ} \mathrm{C}$. At this layer thickness, the InAs critical layer thickness is exceeded and hence the InAs growth changes to the three-dimensional (3D) mode. The SAD's were then capped by a GaAs layer grown at $630{ }^{\circ} \mathrm{C}$. Within this top layer, an $\mathrm{Al}_{x} \mathrm{Ga}_{1-x} \mathrm{As}$ window is inserted to prevent any surface electrical field. Considering the photoluminescence data and calculations, the size of the dots is determined to be from 70 to $120 \AA$ at the base and from 25 to $40 \AA$ high.

\section{B. The phonon bottleneck observed in cw PL experiments}

The photoluminescence experiments are performed at $T$ $=5 \mathrm{~K}$ with a Ti-sapphire laser pumped by an $\mathrm{Ar}^{+}$laser. The 


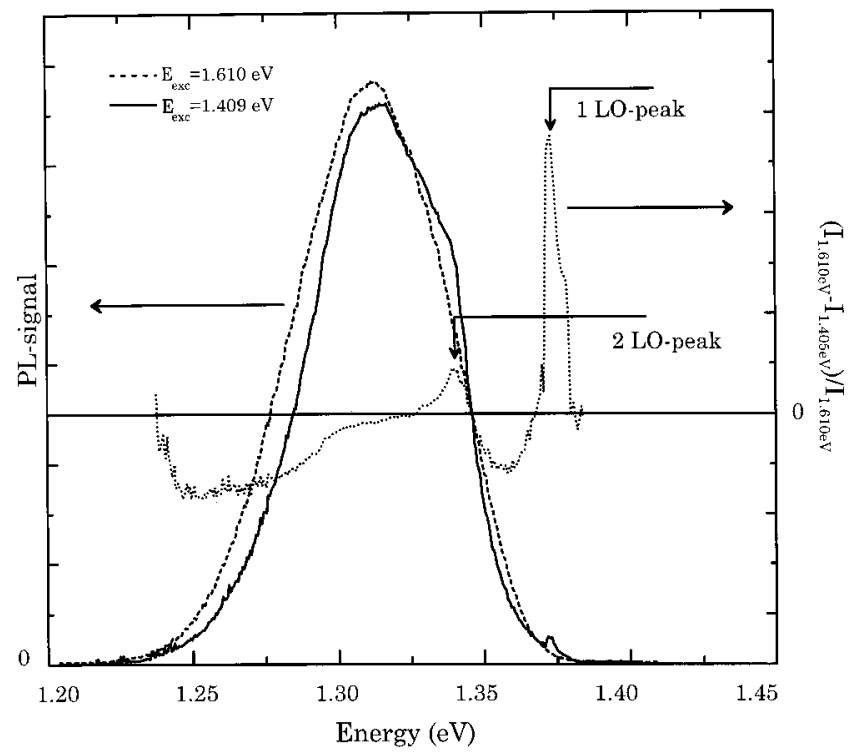

FIG. 1. Photoluminescence spectrum under nonresonant excitation at $1.610 \mathrm{eV}$ (short dashed line) and when exciting one GaAs LO-phonon energy $(1.409 \mathrm{eV})$ above the ground state of the smallest dots (solid line).

signal is collected through a $0.75-\mathrm{m}$ double monochromator on an $\mathrm{InGaAs}(\mathrm{Cs})$ photomultiplier in photon counting mode. For nonresonant PL measurements, the laser is tuned above the GaAs band-gap energy $(1.61 \mathrm{eV})$. This allows for carrier thermalization inside the wetting layer and subsequent capture into SAD's of different sizes.

The spectrum is shown in Fig. 1 (short dashed line). The full width at half maximum (FWHM) of $\pm 65 \mathrm{meV}$ is due to the size distribution of the dots, although a direct link is not evident, since for larger dots nonradiative centers can be formed due to strain relaxation. The reason that all the dots can be accessed in spite of their $\delta$-like density of states (DOS) is that there are enough levels below the excitation energy through which carriers can relax before being captured into the dots. Therefore, the PL line shape does not change even for excitation into the wetting layer provided it is kept far above the edge of the wetting layer at $\pm 1.345 \mathrm{eV}$ according to our PL excitation measurement.

However, close to the edge of the density of states in the wetting layer, the number of states below the excitation energy is limited, as is the number of dots that can be populated efficiently. If the excitation energy is changed to (less than) one GaAs LO-phonon energy above the ground state of the smallest dots, i.e., $1.409 \mathrm{eV}$ in our experiments, the luminescence band starts to change clearly (Fig. 1, solid line).

A suppression of the luminescence is observed between one and two GaAs-like LO-phonon energies below the excitation energy and at the lower-energy side of the PL band. To make this feature clearly visible, the normalized difference between the luminescence when exciting at $1.610 \mathrm{eV}$ and the luminescence when exciting at $1.409 \mathrm{eV}$ is also shown in Fig. 1 (dotted line). It is obvious from these spectra that the presence of the phonon bottleneck can be observed without performing time-resolved measurements. Only the

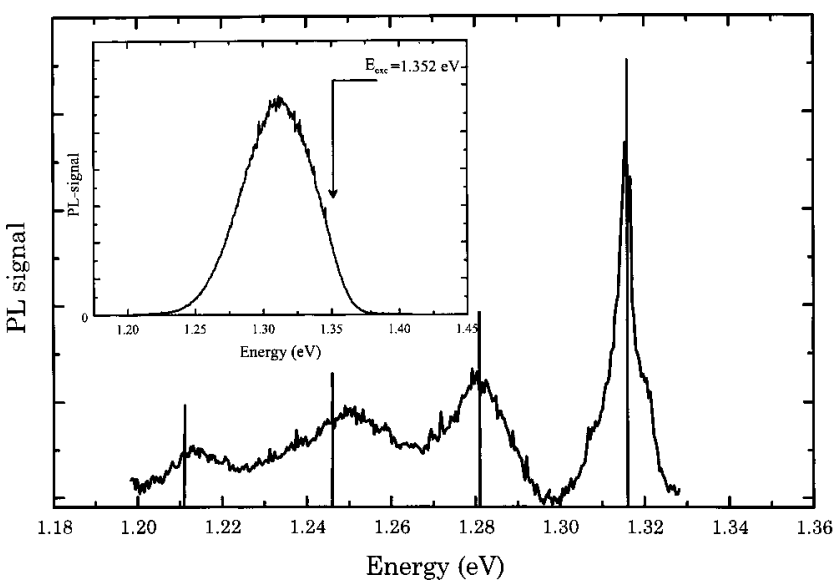

FIG. 2. Photoluminescence spectrum under resonant excitation at $1.352 \mathrm{eV}$. The vertical bars are separated by $35 \mathrm{meV}$.

dots with the ground-state energy equal to the excitation energy minus once or twice the GaAs-like LO-phonon energy are populated efficiently. Their $\delta$-like DOS can be accessed directly from the excited energy level in the wetting layer by LO-phonon emission.

\section{Multiphonon emission by Fröhlich interaction}

To overcome the phonon bottleneck, the dots are excited resonantly, i.e., exciting a given dot size with the groundstate energy fitting to the excitation energy. A spectrum observed under such resonant excitation at $1.352 \mathrm{eV}$ is shown in Fig. 2. The inhomogeneously broadened PL band (see the inset of Fig. 2) is now developed into four phonon-emission enhanced bands. All the bands are evenly spaced by $35 \mathrm{meV}$ (GaAs-like LO phonon). It should be mentioned that the third peak is off its ideal position by $2 \mathrm{meV}$. As three phonons are involved, in the energy scale this corresponds to 2\% departure. Our recent micro-PL (Ref. 12) experiments show that this deviation might be attributed to the spread in the phonon energies $( \pm 1.5 \mathrm{meV})$ due to the (nonuniform) strain in the dots. The first band at $35 \mathrm{meV}$ is only sharp if it appears at the spectral position of the small dots. ${ }^{9}$ This is attributed to an enhanced contribution of the surrounding GaAs matrix in the relaxation process of the smallest dots due to a higher leakage of the wave functions into the GaAs. The FWHM of the three other bands is $\pm 12 \mathrm{meV}$, which is much larger than the homogeneous broadening. We assume two possible explanations for the extra broadening of these peaks. First, as reported in Ref. 7, even a single quantum dot exhibits a continuous density of states. Under selective size excitation, this translates into inhomogeneous broadening due to the size distribution. Second, Pekar's formula is not obeyed in our experiments; that is, nonadiabaticity further broadens the phonon-emission assisted bands. The phononemission enhanced bands are, as mentioned above, also reported for larger dots. However, since the PL measurements at high excitation density show no excited states, the intradot electronic transitions are not possible in such small dots. We propose an enhanced phonon emission by the dots due to strong electron-phonon coupling. 


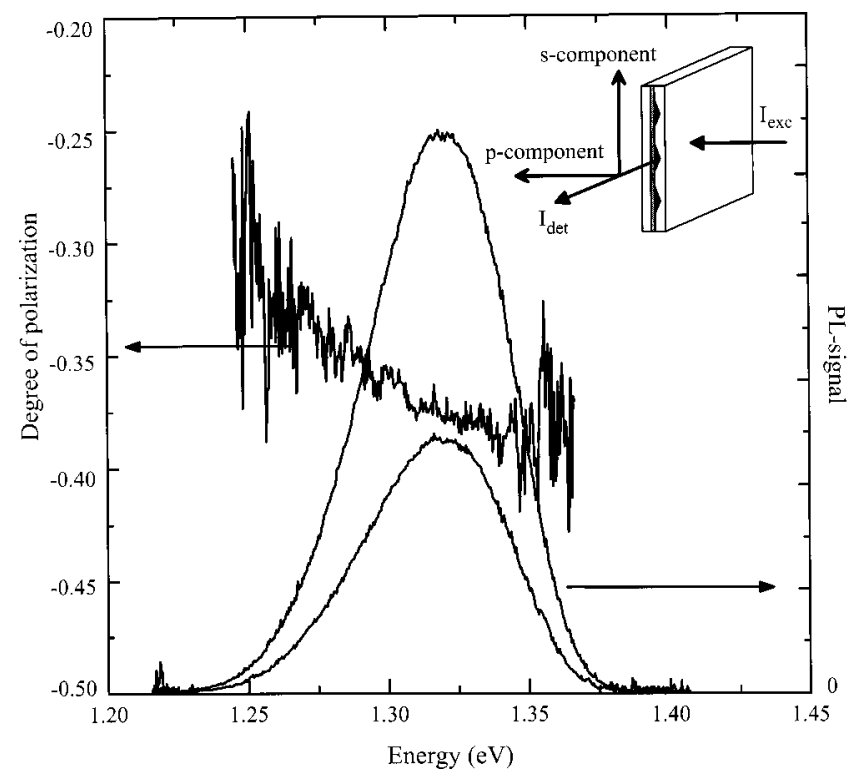

FIG. 3. The cleaved-side (see inset) polarization-dependent photoluminescence spectra under nonresonant excitation at $1.601 \mathrm{eV}$.

Two possible electron-phonon interactions can be present in the dots: Fröhlich or polar interaction and deformation coupling. ${ }^{13}$ We focus on the polar interaction since no transverse optical (TO) modes are observed in our cleaved-side measurements, which is a clear indication that we are dealing with Fröhlich interaction only. Additionally, since none of the Raman selection rules is obeyed, either in backscattering and cleaved-side geometries, we excluded possible contributions from the Raman scattering. A localized polarization gives rise to an enhancement of the Fröhlich interaction as observed in $\mathrm{In}_{x} \mathrm{Ga}_{1-x} \mathrm{As} / \mathrm{InP}$ quantum wells. ${ }^{14}$ After recombination of the photocarriers, depolarization takes place and the stored electrostatic energy is released by emitting LO phonons. In such a process, no real crystal states are involved.

This requires that the electrons and holes are localized differently in the dots. The nonuniform strain field present in the dots, ${ }^{4-6,11}$ even during the formation, ${ }^{15}$ results in such a different localization, with the hole more confined inside the dot and more compact than the electrons. ${ }^{5}$ The net charge distribution is orientated in the growth direction, according to the envelope functions reported in Refs. 4, 5, and 11. Besides the discussed calculations, such a permanent dipole is observed in recent photocurrent measurements on quantum dots. ${ }^{16}$

\section{Polarization-dependent photoluminescence experiments}

The polarization of the dipole allowed transitions has been discussed theoretically. ${ }^{4,5,11}$ For the holes confined at the base of the SAD's, one should observe $s$ polarization of the ground-state optical transition. To compare the theoretical expected polarization with experiments, nonresonant cleaved-side photoluminescence measurements were performed.

The results are shown in Fig. 3. The degree of polariza-

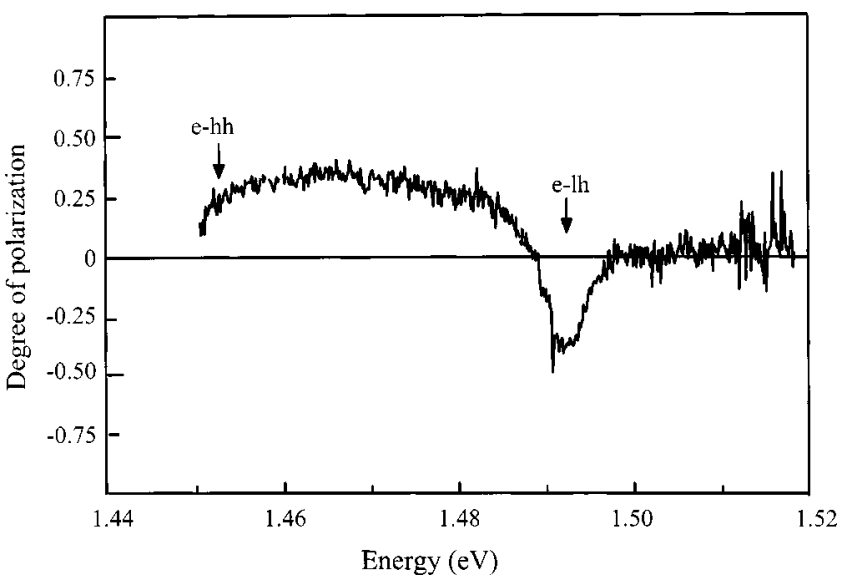

FIG. 4. Cleaved-side polarization-dependent PL excitation measurements on the InAs/GaAs thin quantum-well structure as described in Ref. 15.

tion indicated on the left axis is defined as $\left(I_{s}-I_{p}\right) /\left(I_{s}\right.$ $+I_{p}$ ), where $I_{s}$ is the intensity of the luminescence perpendicular to the growth direction and $I_{p}$ is the intensity of the luminescence in the growth direction. Experimentally, we observe $p$, not $s$, polarization of more than $30 \%$.

To illustrate this difference between the expected and observed polarization for the dots, we compare the observed polarization with the cleaved-side excitation measurements on the InAs monolayer structures. ${ }^{17}$ In compressivelly strained thin InAs/GaAs quantum wells, we measure $s$ polarization as it is expected for the ground state. The degree of polarization is shown in Fig. 4. The $p$ polarization on the higher-energy side of the spectrum is due to the excited state, which is the light hole.

An elongation in the structure of the dots could be an explanation for the discrepancy between the theoretically predicted and the experimentally observed polarization. This elongation introduces an optical anisotropy, for which the intensity of the luminescence is higher in the direction of the elongation compared to the intensity perpendicular to it. Submonolayers epitaxy of InAs on (001) GaAs (Ref. 18) and InAs on high indexed GaAs (Ref. 19) show this optical anisotropy. However, according to the observed $p$ polarization, the elongation has to be directed in the growth direction, which is, considering the shape of the dots discussed here, not very likely. Nevertheless, the orientation of the facets, without introducing an elongation, could influence the dipole allowed transitions and thus the polarization of the groundstate luminescence, as is mentioned in Ref. 5. An assumption of the calculations in Refs. 4, 5, and 11, which is certainly worth mentioning with respect to the polarization, is the neglect of the spin-orbit coupling. It is known from calculations on CdTe nanocrystals ${ }^{20}$ that the incorporation of the spin-orbit coupling has a large impact on the optically allowed transition.

According to Ref. 16, the permanent electrical dipole is antiparallel to the theoretical calculated charge distribution. This means that the holes should be localized at the apex of the InAs/GaAs SAD's. Our experimental value of the polar- 
ization confirms this picture. The nonuniform strain in the dots induced ${ }^{4,5}$ favors the light-hole character of the ground state, provided that the holes are confined close to the apex. It is clear from the reasoning above that there is still an ongoing discussion about the polarization of the dipole allowed transitions. Nevertheless, considering the experimental data, which are carefully checked, we conclude that the ground state in the dots discussed here is light-hole-like.

To summarize, the phonon bottleneck is observed directly from cw-photoluminescence experiments. The phonon emission by the dots does not involve any real crystal states. They are due to enhanced Fröhlich interaction between a straininduced polarized exciton in the dots and LO phonons. We measured the cleaved-side polarization, which demonstrates a light-hole ground state.
Note added. After submission of the manuscript, we learned of the independent, related measurements of Heitz et al. ${ }^{10}$

\section{ACKNOWLEDGMENTS}

We wish to acknowledge that this work is part of the research program of the "Stichting Fundamenteel Onderzoek der Materie (FOM)," which is financially supported by the "Nederlandse Organisatie voor Wetenschappelijk Onderzoek (NWO)." We would also like to thank A. GarciaCristobal (Universidad de Valencia) and V. M. Fomin and J. T. Devreese [University of Antwerp (UVA)] for fruitful discussions, J. Brübach for the cleaved-side measurements on the InAs-ML structures, and M. S. Skolnick for discussing their photocurrent measurement prior to publication.
*Present address: ASM-Lithography SA, De Run 1110, 5503 LA Veldhoven, The Netherlands.

†Author to whom correspondence should be addressed; E mail: A.Silov@phys.tue.nl

Deceased.

${ }^{1}$ R. Heitz et al., Appl. Phys. Lett. 68, 361 (1996).

${ }^{2}$ S. Fafard et al., Phys. Rev. B 52, 5752 (1995).

${ }^{3}$ M. J. Steer et al., Phys. Rev. B 54, 17738 (1996).

${ }^{4}$ M. A. Cusack, P. R. Briddon, and M. Jaros, Phys. Rev. B 56, 4047 (1997).

${ }^{5}$ J. Kim, L.-W. Wang, and A. Zunger, Phys. Rev. B 57, R9408 (1998).

${ }^{6}$ C. Pryor et al., J. Appl. Phys. 83, 2548 (1998).

${ }^{7}$ Y. Toda, O. Moriwaki, M. Nishioka, and Y. Arakawa, Phys. Rev. Lett. 82, 4114 (1999).

${ }^{8}$ J.-Y. Marzin and G. Bastard, Solid State Commun. 92, 437 (1994).

${ }^{9}$ A. W. E. Minnaert et al., in Proceedings of the International Conference on the Physics of Semiconductors, Jerusalem, 1998, edited by D. Gershoni (World Scientific, Singapore, 1999) (on
CD), Sec. VII. One- and Zero-Dimensional Systems, Subsection B: Optical Studies of Wires and Dots, No. 36.

${ }^{10}$ R. Heitz et al. Phys. Rev. Lett. 83, 4654 (1999).

${ }^{11}$ M. Grundmann, O. Stier, and D. Bimberg, Phys. Rev. B 52, 11969 (1995).

${ }^{12}$ A. W. E. Minnaert, A. Yu. Silov, and J. H. Wolter, in Proceedings of the International Conference on the Physics of Semiconductors, Osaka, 2000 (to be published), abstract D192.

${ }^{13}$ A. V. Fedorov, A. V. Baranov, and K. Inoue, Phys. Rev. B 56, 7491 (1997).

${ }^{14}$ K. J. Nash, M. S. Skolnick, P. A. Claxton, and J. S. Roberts, Phys. Rev. B 39, 5558 (1989).

${ }^{15}$ P. M. Petrof and G. Medeiros-Ribeiro, MRS Bull. April, 50 (1996).

${ }^{16}$ W. Fry et al., Phys. Rev. Lett. 84, 733 (2000).

${ }^{17}$ J. Brübach et al., Phys. Rev. B 59, 10315 (1999).

${ }^{18}$ P. D. Wang et al., Appl. Phys. Lett. 64, 1526 (1994).

${ }^{19}$ M. Henini et al., Phys. Rev. B 57, R6815 (1998).

${ }^{20}$ P. Lefebvre, T. Richard, H. Mathieu, and J. Allgère, Solid State Commun. 98, 303 (1996). 